\title{
Floquet engineering bulk odd-frequency superconducting pairs
}

\author{
Jorge Cayao, ${ }^{1}$ Christopher Triola, ${ }^{2,1}$ and Annica M. Black-Schaffer ${ }^{1}$ \\ ${ }^{1}$ Department of Physics and Astronomy, Uppsala University, Box 516, S-751 20 Uppsala, Sweden \\ ${ }^{2}$ Los Alamos National Laboratory, Los Alamos, New Mexico 87544, USA
}

(Received 17 August 2020; revised 9 February 2021; accepted 25 February 2021; published 8 March 2021; corrected 22 April 2021)

\begin{abstract}
We introduce the concept of Floquet odd-frequency superconducting pairs and establish their emergence in time-periodic conventional superconductors. We show that these exotic Cooper pairs are possible because the Floquet modes in time-periodic systems provide an additional index (a Floquet index) that broadens the classification of superconducting pair symmetries, with no analog in the static regime. Our results thus put forward a different route for odd-frequency superconducting pairs, and pave the way for Floquet engineered dynamical superconducting states.
\end{abstract}

DOI: 10.1103/PhysRevB.103.104505

\section{INTRODUCTION}

Since its discovery, superconductivity has garnered widespread attention not only owing to its fundamental mechanisms but also due to its large number of applications, making it one of the core areas in condensed matter. Recent developments have reported a plethora of remarkable superconducting states, such as chiral superconductors [1], hightemperature superconductors [2], topological superconductors [3], superconducting metamaterials [4], magic angle superconductors [5], and nematic superconductors [6]. This vast diversity of superconductors is highly reliant on the symmetries of their fundamental building blocks, the Cooper pairs.

The symmetries of Cooper pairs are constrained by the fermionic nature of the constituent electrons [7], which imposes antisymmetry on the Cooper pair amplitude under the interchange of all quantum numbers of the paired electrons, including the exchange of their time coordinates. This allows for the usual pair correlations between electronic states at equal times, but also permits electron pairing at different times. Remarkably, such unequal time pairing enables the emergence of Cooper pairs with a pair amplitude that is odd under the exchange of time coordinates or, equivalently, odd in frequency [8-15]. Since odd-frequency (odd- $\omega$ ) pair amplitudes vanish at equal times, this type of pairing is intrinsically nonlocal in time and represents a truly dynamical effect.

The emergence of odd- $\omega$ pairs has been shown to be related to the breaking of system symmetries, and a variety of superconducting systems are believed to host these exotic correlations [16-20]. In particular, superconducting heterostructures [11-15,21-25] with experimental observations in ferromagnetic junctions [26-28] and superconductors with multiple degrees of freedom [29-35] are the most notable

Published by the American Physical Society under the terms of the Creative Commons Attribution 4.0 International license. Further distribution of this work must maintain attribution to the author(s) and the published article's title, journal citation, and DOI. Funded by Bibsam. examples. In heterostructures, odd- $\omega$ pairs are induced by the breaking of spatial translation symmetry, while in multiband superconductors hybridization between bands, which can be seen as an intrinsic symmetry breaking effect, gives rise to odd- $\omega$ pairs.

Despite the dynamical nature of odd- $\omega$ pairing, the overwhelming majority of previous work has focused on static systems and static properties of odd- $\omega$ pairing. Moreover, the few works which have found odd- $\omega$ pairing induced by timedependent drives have shown that mere time dependence is not sufficient to generate odd- $\omega$ pairing. The drive must either break translation invariance [36] or couple nontrivially to a band index [37]. While time-dependent drives may reduce the order parameter [38,39], e.g., via heating, there are strategies to mitigate this effect, for example, choosing a drive frequency that is large compared with the relevant energy scales in the system [40]. Additionally, external drives have even been shown to enhance or induce superconductivity [41-48].

In this work we show that odd- $\omega$ superconducting pairs are generically present in time-periodic superconductors, where properties are described in terms of Floquet bands (see Fig. 1). We find that these Floquet bands provide an additional index
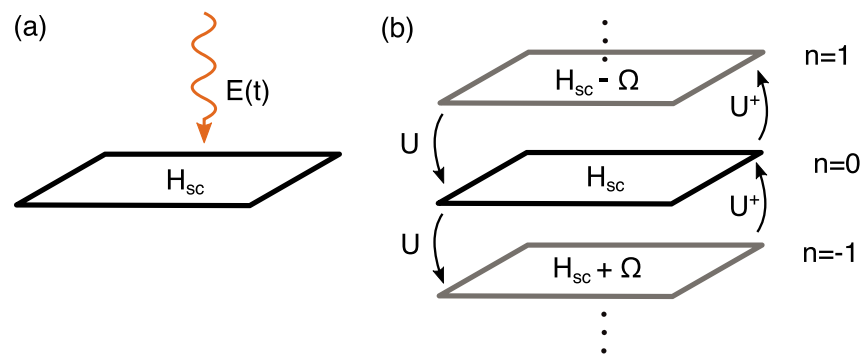

FIG. 1. (a) Sketch of a superconductor, described by a static Hamiltonian $H_{\text {sc }}$ driven by a time-periodic field $E(t)$ with frequency $\Omega$ (wiggle orange arrow). (b) Within Floquet theory the original problem is described in terms of Floquet bands, or sidebands, labeled by the Floquet index $n$, which are replicas of the undriven system $H_{\text {sc }}$ shifted by $n \Omega$ and coupled by $U$ (arc black arrows), which depend on $E(t)$. 
that, remarkably, broadens the classification of pair symmetries with no analog in the static regime. In particular, we introduce the concept of Floquet odd- $\omega$ pairing and demonstrate its emergence in conventional (spin-singlet, $s$-wave) superconductors driven by circularly polarized light, where it acquires a large magnitude without any fine-tuning of model parameters, and can be controlled by the drive. The prospect of inducing and controlling dynamical pairs by time-periodic fields opens the route for Floquet engineered dynamical superconducting correlations.

\section{FLOQUET PAIR AMPLITUDES}

To show the emergence of Floquet odd- $\omega$ Cooper pairs, we first provide a general characterization of their symmetries. To begin, we note that the pair amplitudes of any superconductor (driven or static) are given by the anomalous Green's function $[49,50] F^{\sigma_{1}, \sigma_{2}}\left(\boldsymbol{k}_{1}, \boldsymbol{k}_{2} ; t_{1}, t_{2}\right)=-i\left\langle\mathcal{T} c_{\boldsymbol{k}_{1}, \sigma_{1}}\left(t_{1}\right) c_{\boldsymbol{k}_{2}, \sigma_{2}}\left(t_{2}\right)\right\rangle$, where $\mathcal{T}$ is the time-ordering operator and $c_{k, \sigma}(t)$ annihilates an electronic state with spin $\sigma$, momentum $\boldsymbol{k}$, at time $t$. The fermionic nature of electrons then imposes the antisymmetry condition $F^{\sigma_{1}, \sigma_{2}}\left(\boldsymbol{k}_{1}, \boldsymbol{k}_{2} ; t_{1}, t_{2}\right)=-F^{\sigma_{2}, \sigma_{1}}\left(\boldsymbol{k}_{2}, \boldsymbol{k}_{1} ; t_{2}, t_{1}\right)$, which is responsible for the different pair symmetries [18], and in particular allows for pair amplitudes that are odd in $\omega$ when $F^{\sigma_{1}, \sigma_{2}}\left(\boldsymbol{k}_{1}, \boldsymbol{k}_{2} ; t_{1}, t_{2}\right)=-F^{\sigma_{1}, \sigma_{2}}\left(\boldsymbol{k}_{1}, \boldsymbol{k}_{2} ; t_{2}, t_{1}\right)$.

While the antisymmetry condition applies to any two-time pair amplitude [18], we wish to investigate the properties of pair amplitudes in Floquet systems. Therefore, we next consider a superconductor in a time-periodic field $E(t)$ with period $T=2 \pi / \Omega$, as shown in Fig. 1(a). This system is described by a time-dependent Hamiltonian $H(t)$ that inherits the time periodicity of $E(t)$, such that $H(t)$ $=H(t+T)$. For such a time-periodic Hamiltonian, the Floquet theorem [51-53] allows us to decompose the solutions to the Schrödinger equation in terms of harmonics of the fundamental drive frequency $\Omega$. Similarly, we decompose the anomalous Green's function as [54],

$$
\begin{aligned}
& F^{\sigma_{1}, \sigma_{2}}\left(\boldsymbol{k}_{1}, \boldsymbol{k}_{2} ; t_{1}, t_{2}\right) \\
& \quad=\sum_{m, n} \int \frac{d \omega}{2 \pi} \mathrm{e}^{-i(\omega+n \Omega) t_{1}+i(\omega+m \Omega) t_{2}} F_{n, m}^{\sigma_{1}, \sigma_{2}}\left(\boldsymbol{k}_{1}, \boldsymbol{k}_{2}, \omega\right),
\end{aligned}
$$

where the coefficients $F_{n, m}$ represent the Floquet pair amplitudes, labeled by the Floquet indices $n, m \in \mathbb{Z}$, and $\omega \in$ $[-\Omega / 2, \Omega / 2]$. For details see the Supplementary Material (SM) [55]. Noting that the symmetries of the quantity on the left-hand side of Eq. (1) are constrained by the fermonic antisymmetry condition, we obtain a constraint for the symmetries of the Floquet modes, $F_{n, m}$,

$$
F_{n, m}^{\sigma_{1}, \sigma_{2}}\left(\boldsymbol{k}_{1}, \boldsymbol{k}_{2} ; \omega\right)=-F_{-m,-n}^{\sigma_{2}, \sigma_{1}}\left(\boldsymbol{k}_{2}, \boldsymbol{k}_{1} ;-\omega\right) .
$$

Here, the exchange in Floquet indices, $(n, m) \rightarrow(-m,-n)$, stems from the Floquet decomposition in Eq. (1), intrinsic in two-time periodic functions [54].

From the constraint in Eq. (2) we get all possible Floquet pair symmetries that can emerge in a time-periodic superconducting system, as enumerated in Table I. Remarkably, there are four different classes of odd- $\omega$ pairs, determined by a combination of the Floquet index, spin (singlet, triplet), and momentum ( $s$-wave, $p$-wave etc.). At first sight, it might
TABLE I. All possible symmetries for Floquet pair amplitudes allowed by the antisymmetry condition Eq. (2).

\begin{tabular}{ccccc}
\hline \hline$\frac{0}{0}$ & Floquet index & Frequency & Spin & Momentum \\
& $(n, m) \rightarrow(-m,-n)$ & $\omega \rightarrow-\omega$ & $s= \pm 1$ & $\boldsymbol{k}_{1} \rightarrow \boldsymbol{k}_{2}$ \\
\hline 1 & Even & Even & Singlet & Even \\
2 & Even & Even & Triplet & Odd \\
3 & Odd & Even & Singlet & Odd \\
4 & Odd & Even & Triplet & Even \\
5 & Even & Odd & Triplet & Even \\
6 & Even & Odd & Singlet & Odd \\
7 & Odd & Odd & Triplet & Odd \\
8 & Odd & Odd & Singlet & Even \\
\hline \hline
\end{tabular}

appear that the Floquet indices $(n, m)$ simply act as a new kind of band index, and thus Eq. (2) is just a generalization of the antisymmetry constraint for a multiband superconductor [20] with an arbitrarily large number of band degrees of freedom. To show that this is in fact not the case, we note that the antisymmetry condition for a two-band superconductor is given by $F_{\alpha, \beta}^{\sigma_{1}, \sigma_{2}}\left(\boldsymbol{k}_{1}, \boldsymbol{k}_{2} ; \omega\right)=-F_{\beta, \alpha}^{\sigma_{2}, \sigma_{1}}\left(\boldsymbol{k}_{2}, \boldsymbol{k}_{1} ;-\omega\right)$, where $\alpha$ and $\beta$ are the band indices [20]. Thus, the Floquet symmetry constraint, $(n, m) \rightarrow(-m,-n)$, is very different from that of a multiband superconductor. Crucially, this implies that the Floquet pair amplitudes $F_{n, m}$ can in general develop even and odd terms in the Floquet index, which can both exhibit even- $\omega$ or odd- $\omega$ dependence. Yet another difference with the multiband case is that $n, m \in \mathbb{Z}$, implying that $n, m$ can also be negative. Here, inter-Floquet-band amplitudes $F_{n,-n}$ must be even in the Floquet index, while intra-Floquet-band amplitudes $F_{n, n}$ can develop both even and odd terms in the Floquet index for $n \neq 0$, which remarkably can be both even$\omega$ or odd $-\omega$. This is in stark contrast to the case of multiband systems in which only the interband pairing can be odd in the band index, and thus odd in frequency for even-parity, spinsinglet superconductors. Hence, the Floquet pair amplitudes are unique and exhibit symmetry classes with no analog in undriven systems.

\section{REALIZATION OF FLOQUET ODD- $\omega$ PAIRS}

Next we show the emergence of the Floquet pair amplitudes $F_{n, m}$ discussed above. We consider a simple model that both captures the essential physics of the problem and is easy to implement experimentally: an electronic system possessing a spin-singlet $s$-wave superconducting order parameter $\Delta$ driven by a circularly-polarized light field $\mathbf{E}(t)[56,57]$. Superconductivity could be realized using a conventional superconductor, or could be engineered using proximity effects in heterostructures for additional control of $\Delta[58,59]$. The superconductor is modeled by $H_{\mathrm{sc}}=\xi_{\boldsymbol{k}} \tau_{z}+\Delta \tau_{x}$ in Nambu space $\Psi_{\boldsymbol{k}}(t)=\left[c_{\boldsymbol{k}, \uparrow}(t), c_{-\boldsymbol{k}, \downarrow}^{\dagger}(t)\right]^{\mathrm{T}}$, where $\xi_{\boldsymbol{k}}=\boldsymbol{k}^{2} / 2 m-\mu$ is the kinetic energy with $\boldsymbol{k}=\left(k_{x}, k_{y}, k_{z}\right), m$ the effective mass, $\mu$ the chemical potential, $\Delta$ considered to be an input parameter, and $\tau_{i}$ the $i$-Pauli matrix in Nambu space. The effect of $\mathbf{E}(t)$ is taken into account via the minimal coupling $\boldsymbol{k} \rightarrow \boldsymbol{k}+e \mathbf{A}(t)$, where $\mathbf{A}(t)$ is the vector potential, $\mathbf{E}(t)=$ $-\partial_{t} \mathbf{A}(t)$, and $e>0$ the elementary charge. We take $\mathbf{A}(t)=$ 
$A_{0}(-\sin \Omega t, \sigma \cos \Omega t, 0)$ with period $T=2 \pi / \Omega$, where $\sigma=$ \pm 1 denotes left-/right-handed polarizations and $\Omega$ the frequency of the light. Then, if we redefine $\mu$ as $\mu \rightarrow$ $\mu-e^{2} A_{0}^{2} /(2 m)$, the time-dependent Hamiltonian takes the form $H_{k}(t)=H_{\mathrm{sc}}+V_{\boldsymbol{k}}(t)$ with $V_{\boldsymbol{k}}(t)=(e / m) \mathbf{A}(t) \cdot \boldsymbol{k} \tau_{0}$ a periodic function in $t$.

We are interested in the pair amplitudes of the timeperiodic superconducting system $H_{k}(t)$. These are the electron-hole component of the Nambu space Green's function $\hat{\mathcal{G}}\left(\boldsymbol{k} ; t_{1}, t_{2}\right)=-i\left\langle\mathcal{T} \Psi_{\boldsymbol{k}}\left(t_{1}\right) \Psi_{\boldsymbol{k}}^{\dagger}\left(t_{2}\right)\right\rangle$, obtained by solving the equation of motion $\left[i \partial_{t_{1}}-H_{\boldsymbol{k}}\left(t_{1}\right)\right] \hat{\mathcal{G}}\left(\boldsymbol{k} ; t_{1}, t_{2}\right)=\delta\left(t_{1}-t_{2}\right)$. Given the time periodicity of the Hamiltonian $H_{k}(t)$, we can decompose $\hat{\mathcal{G}}$ in terms of Floquet modes [51-53] and write the equation of motion for $\hat{\mathcal{G}}$ as [54]

$$
\sum_{m^{\prime}}\left[\omega \delta_{n, m^{\prime}}-H_{n, m^{\prime}}\right] \mathcal{G}_{m^{\prime}, m}(\boldsymbol{k}, \omega)=\delta_{n, m},
$$

where $\omega \in[-\Omega / 2, \Omega / 2]$ and $H_{n, m^{\prime}}=\left(H_{\mathrm{sc}}-m^{\prime} \Omega\right) \delta_{n, m^{\prime}}+$ $U_{\boldsymbol{k}} \delta_{n+1, m^{\prime}}+U_{\boldsymbol{k}}^{*} \delta_{n-1, m^{\prime}}$ is the Floquet Hamiltonian. Here $U_{k}=(1 / T) \int_{0}^{T} d t \mathrm{e}^{i \Omega t} V_{k}(t)=e A_{0} /(2 m)\left(\sigma k_{y}-i k_{x}\right) \tau_{0}$

couples nearest-neighbor sidebands, which involves the emission/absorption of a photon. Finally, the Floquet pair amplitudes $F_{n, m}$ are given by the off-diagonal elements of the Floquet Green's function $\mathcal{G}_{n, m}$, see SM [55]. Note that spin rotation symmetry is preserved such that all pair amplitudes must be spin singlet.

The sum over Floquet modes in Eq. (3) runs in principle to infinity, but since we focus on a finite range of $\omega$, a truncation of this sum approximates the exact answer well $[40,54,60]$. For instructive purposes, we first restrict our attention to modes with $n, m \in\{-1,0,1\}$. Even though $\mathcal{G}$ can be directly found from Eq. (3), to visualize its functional dependences we consider the limit $U_{k} / \Omega \ll 1$ and expand the Dyson's equation to second order in $U_{k} / \Omega$. Here we show the main findings, while details are found in the SM [55]. We find Floquet pair amplitudes of the form $F_{n, m}$ with $n, m \in\{-1,0,1\}$, and for a better analysis we decompose them into their even and odd terms in Floquet indices as $F_{n, m}^{ \pm}=\left(F_{n, m} \pm F_{-m,-n}\right) / 2$, obtaining all nonzero amplitudes,

$$
\begin{aligned}
F_{0,0}^{+}(\boldsymbol{k}, \omega) & \approx \frac{\Delta}{D}+\frac{\Delta\left|U_{k}\right|^{2} A_{\omega}^{\Omega}}{D^{2} D_{-1} D_{1}}, \\
F_{1,1}^{+}(\boldsymbol{k}, \omega) & \approx \frac{\Delta\left[D+\Omega^{2}\right]}{D_{1} D_{-1}}+\frac{\Delta\left|U_{k}\right|^{2} B_{\omega}^{\Omega}}{2 D\left(D_{-1} D_{1}\right)^{2}}, \\
F_{1,1}^{-}(\boldsymbol{k}, \omega) & \approx \frac{-2 \omega \Omega \Delta}{D_{1} D_{-1}}+\frac{2 \omega \Omega \Delta\left|U_{k}\right|^{2} C_{\omega}^{\Omega}}{D\left(D_{-1} D_{1}\right)^{2}}, \\
F_{1,-1}^{+}(\boldsymbol{k}, \omega) & \approx-\frac{\Delta\left[U_{k}^{*}\right]^{2} E_{\omega}^{\Omega}}{D D_{1} D_{-1}}, \\
F_{0,1}^{+}(\boldsymbol{k}, \omega) & \approx-\frac{2 \omega \Delta U_{k}}{D_{1} D_{-1}}, \\
F_{0,1}^{-}(\boldsymbol{k}, \omega) & \approx-\frac{\Omega \Delta U_{k} E_{\omega}^{\Omega}}{D D_{1} D_{-1}},
\end{aligned}
$$

where $D(\omega)=\omega^{2}-\left(\Delta^{2}+\xi_{k}^{2}\right)$ and $D_{n}(\omega)=D(\omega+n \Omega)$. Here, $A_{\omega}^{\Omega}, B_{\omega}^{\Omega}, C_{\omega}^{\Omega}, E_{\omega}^{\Omega}$, and $D_{1} D_{-1}$ are even functions of $\boldsymbol{k}$ and $\omega$, whose explicit expressions are not necessary for our
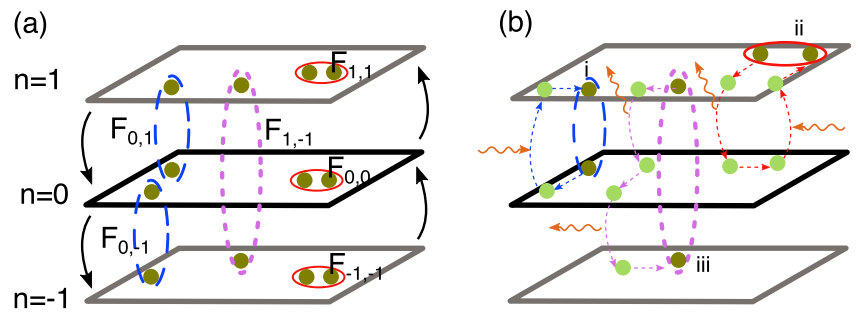

FIG. 2. (a) Schematics of the intra- and intersideband Floquet pairs, where black arrows represent the couplings between sidebands. (b) Formation of the pairs in (a) involve bare and dressed processes due to the coupling between sidebands, shown here for (i) $F_{0,1}$ (blue), (ii) $F_{1,1}$ (red), and (iii) $F_{1,-1}$ (magenta), with absorption/emission of photons (orange wiggle arrows).

discussion but are given in the SM [55]. The first three expressions in Eqs. (4) describe Cooper pairs that emerge within each sideband (intrasideband), while the last three describe pairs between electrons in different sidebands (intersideband), as depicted in Fig. 2(a). All these pairs contain both bare and dressed processes due to the coupling between sidebands via $U_{k}$, shown in Fig. 2(b) for three illustrative cases, which involve absorption/emission of photons (orange wiggle arrows).

The intrasideband amplitudes, $F_{0,0}^{+}$and $F_{1,1}^{ \pm}$in Eqs. (4), include bare contributions (first term on the right-hand side) and corrections proportional to $\left|U_{k}\right|^{2}$, that involve transitions between sidebands assisted by two-photon (emission and absorption of a photon) processes, see, e.g., process (ii) in Fig. 2(b) and the SM [55]. We verify that higher order corrections always require an even number of photons. The amplitude for $n=m=0$ is purely even in Floquet indices $F_{0,0}^{+}$, even- $\omega$, and even in $\boldsymbol{k}$, thus belonging to symmetry class 1 in Table I. Interestingly, we find that $n, m= \pm 1$ pair amplitudes develop both even and odd terms in Floquet indices, $F_{1,1}^{ \pm}$. Here $F_{1,1}^{+}$is even in both frequency and momentum, thus belonging to class 1 in Table I, while $F_{1,1}^{-}$instead clearly has an odd- $\omega$ dependence even at zeroth order in $U_{k}$, which is directly controllable by the drive frequency $\Omega$. We note that $F_{1,1}^{-}$is also odd in the Floquet indices but even in momentum and thus belongs to symmetry class 8 in Table I. These results highlight a key aspect: the characterization of pair symmetries of periodically-driven superconducting states of matter is unique and intrinsically different from the equilibrium case and can be induced and controlled by time-periodic fields.

In contrast to the intrasideband case, the intersideband pair amplitudes in Eqs. (4) always require transitions between sidebands (via $U_{k}$ ) and thus involve absorption or emission of photons. We distinguish between two types of pair amplitudes, requiring nearest-neighbor (e.g., $F_{0,1}^{ \pm}$between $n=0$ and $n=1$ ) or next-nearest-neighbor transitions (e.g., $F_{1,-1}^{+}$). Here, $F_{0,1}^{ \pm}$is linear in $U_{k}$ and only necessitates a one-photon process as depicted in process (i) Fig. 2(b). However, $F_{1,-1}^{+}$is proportional to $\left[U_{k}^{*}\right]^{2}$ and thus needs two-photon processes of the same kind, either absorption or emission of two photons, as depicted in process (iii) Fig. 2(b). Note this two-photon process is still fundamentally different from the intrasideband amplitudes. Here we find that only the even Floquet index 
component $F_{0,1}^{+}$develops an odd- $\omega$ term which is proportional to $\omega$, instead of $\omega \Omega$ found for intrasideband terms. Surprisingly, it is also odd in momentum $\boldsymbol{k}$ (class 6 in Table I) and thus of $p$-wave nature, despite the $s$-wave symmetry of the superconductor. This is a consequence of the linear coupling between the light field and momentum. Finally, we find that $F_{0,1}^{-}$and $F_{1,-1}^{+}$belong to classes 3 and 1, respectively, in Table I.

After analytically confirming the emergence of Floquet pair amplitudes $F_{n, m}$ within perturbation theory, we proceed to compare the relative sizes of each of the symmetry classes by solving Eq. (3) numerically to infinite order. For this purpose we truncate the sum over the Floquet indices, such that $n, m \in[-N, N]$ for some integer cutoff $N$, and numerically obtain $\mathcal{G}$, whose anomalous components then yield $F_{n, m}$. Finally, we decompose $F_{n, m}$ into the various symmetry classes of Table I and find finite amplitudes belonging to classes 1, 3, 6, and 8, shown in Fig. 3 as a function of $\omega$ and $\boldsymbol{k}$. We verify that the overall physical dependencies on $\omega$ and $\boldsymbol{k}$ of these amplitudes are consistent with the second order results given by Eqs. (4) and that all other symmetry classes are equal to zero, supporting the validity of the perturbation approach used above. We have also checked that the results of Fig. 3 remain unchanged for larger values of $N$ and do not depend sensitively on the choice of model parameters.

The bright areas of the pair amplitudes in Fig. 3 extend to higher $\omega$ and reflect the fact that each pair amplitude contains the contribution from many Floquet components. Indeed, in all panels we observe the energy versus momentum dispersion Floquet replicas, a standard feature of Floquet superconductors. These Floquet replicas can be measured, e.g., by time- and angle-resolved photoemission spectroscopy [56,61]. Since the pair amplitudes exist only when the Floquet replicas are present, the experimental observation of these replicas constitutes an indirect but strong sign of the emergence of Floquet pair amplitudes. Note that the even- $\omega$ $(a, d)$ and odd- $\omega$ classes $(b, c)$ exhibit high and low intensities, respectively, near $\omega=0$, thus enabling their distinction. This can be understood by noting that effects at low $\omega$ stem from the lowest Floquet mode $n=0[40,54,60]$, and that $F_{0,0}^{+}$has even- $\omega$ symmetry, see Eqs. (4). At finite frequencies (e.g., at $\omega \approx \Omega$ ) the odd- $\omega$ amplitudes also acquire large values due to individual odd- $\omega$ contributions from higher Floquet modes, e.g., $F_{1,1}^{-}$in Eqs. (4). Moreover, Fig. 3 shows that the even- $\omega$ and odd- $\omega$ amplitudes are of the same order of magnitude, an unusual feature for odd- $\omega$ correlations in conventional superconductors [18].

From the analytical and numerical results presented above, we see that circularly polarized light applied to a conventional $s$-wave spin-singlet superconductor gives rise to a very rich variety of Floquet pair amplitudes which do not emerge in static systems. In particular, we find substantial odd- $\omega$ pairing which only requires breaking the continuous time-translation invariance in time-periodic superconductors, unlike previous studies which needed to break additional symmetries [36,37]. These results not only provide a fundamental understanding of Cooper pairs in driven superconductors, but we also anticipate that the Floquet pair amplitudes can have important consequences in experimental observables [62]. For example, it has been shown that odd- $\omega$ pairing can induce a paramag-
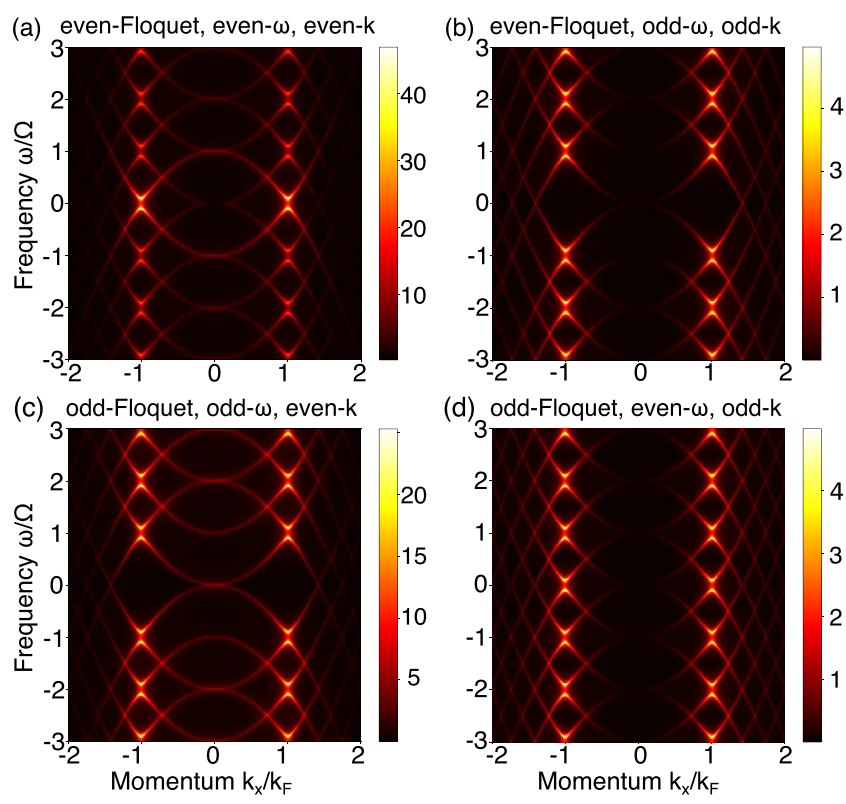

FIG. 3. Floquet pair amplitudes in the $\omega, k_{x}$-plane, projected onto the symmetry classes of Table I: (a) class 1 , (b) class 6 , (c) class 8 , and (d) class 3. Parameters: $\Delta=\Omega / 10, e A_{0} / 2 m=\Omega / 20,2 m=1$, $N=10$.

netic Meissner contribution in multiband systems [28,35,6366]. However, the magnitude of this paramagnetic effect is small due to the small odd- $\omega$ term, and thus is hard to distinguish from the diamagnetic even- $\omega$ contribution. The large Floquet odd- $\omega$ pair amplitudes found here would be expected to remedy that situation. Moreover, our findings might also be relevant for other recent advances in superconductors under time-periodic driving fields, such as Higgs modes in superconductors under radiation [67-69], Floquet Majorana fermions [70,71], and time-crystalline superconductors [72], where the emergence of Floquet odd- $\omega$ pairs should be inevitable and could play an important role.

\section{CONCLUSIONS}

In conclusion, we have demonstrated that the symmetry classification of superconducting pair amplitudes is significantly broadened in time-periodic superconductors by virtue of their Floquet bands. In particular, we have introduced the concept Floquet odd-frequency pair amplitudes with no ana$\log$ in static systems and showed that they can be induced and controlled by the drive frequency even in fully conventional superconductors. The ability to induce and control superconducting pair amplitudes via time-periodic fields [40,73-76] paves the way for Floquet engineering dynamical superconducting states, whose fundamental understanding allow for the design of novel superconducting devices.

\section{ACKNOWLEDGMENTS}

We thank A. Balatsky, D. Chakraborty, F. Parhizgar, and S. Pradhan for useful discussions. We acknowledge financial support from the Swedish Research Council (Vetenskapsrådet Grant No. 2018-03488), the European Research Council (ERC) under the European Unions Horizon 2020 research and 
innovation programme (ERC-2017-StG-757553), the Knut and Alice Wallenberg Foundation through the Wallenberg
Academy Fellows program, and the EU-COST Action CA16218 Nanocohybri.
[1] C. Kallin and J. Berlinsky, Rep. Prog. Phys. 79, 054502 (2016).

[2] A. Drozdov, P. Kong, V. Minkov, S. Besedin, M. Kuzovnikov, S. Mozaffari, L. Balicas, F. Balakirev, D. Graf, V. Prakapenka et al., Nature 569, 528 (2019).

[3] M. Sato and Y. Ando, Rep. Prog. Phys. 80, 076501 (2017).

[4] R. Singh and N. Zheludev, Nat. Photon. 8, 679 (2014).

[5] Y. Cao, V. Fatemi, S. Fang, K. Watanabe, T. Taniguchi, E. Kaxiras, and P. Jarillo-Herrero, Nature 556, 43 (2018).

[6] S. Yonezawa, K. Tajiri, S. Nakata, Y. Nagai, Z. Wang, K. Segawa, Y. Ando, and Y. Maeno, Nat. Phys. 13, 123 (2017).

[7] M. Sigrist and K. Ueda, Rev. Mod. Phys. 63, 239 (1991).

[8] V. L. Berezinskii, Zh. Eksp. Teor. Fiz. 20, 628 (1974) [JETP Lett. 20, 287 (1974).

[9] T. R. Kirkpatrick and D. Belitz, Phys. Rev. Lett. 66, 1533 (1991).

[10] A. Balatsky and E. Abrahams, Phys. Rev. B 45, 13125 (1992).

[11] F. S. Bergeret, A. F. Volkov, and K. B. Efetov, Phys. Rev. Lett. 86, 4096 (2001).

[12] A. Kadigrobov, R. I. Shekhter, and M. Jonson, Europhys. Lett. 54, 394 (2001).

[13] Y. Tanaka, Y. Tanuma, and A. A. Golubov, Phys. Rev. B 76, 054522 (2007).

[14] Y. Tanaka and A. A. Golubov, Phys. Rev. Lett. 98, 037003 (2007).

[15] M. Eschrig, T. Löfwander, T. Champel, J. C. Cuevas, J. Kopu, and G. Schön, J. Low Temp. Phys. 147, 457 (2007).

[16] F. S. Bergeret, A. F. Volkov, and K. B. Efetov, Rev. Mod. Phys. 77, 1321 (2005).

[17] Y. Tanaka, M. Sato, and N. Nagaosa, J. Phys. Soc. Jpn. 81, 011013 (2012).

[18] J. Linder and A. V. Balatsky, Rev. Mod. Phys. 91, 045005 (2019).

[19] J. Cayao, C. Triola, and A. M. Black-Schaffer, Eur. Phys. J. Special Topics 229, 545 (2020).

[20] C. Triola, J. Cayao, and A. M. Black-Schaffer, Ann. Phys. 532, 1900298 (2020).

[21] F. Crépin, P. Burset, and B. Trauzettel, Phys. Rev. B 92, 100507(R) (2015).

[22] J. Cayao and A. M. Black-Schaffer, Phys. Rev. B 96, 155426 (2017).

[23] C. Fleckenstein, N. T. Ziani, and B. Trauzettel, Phys. Rev. B 97, 134523 (2018).

[24] P. Dutta and A. M. Black-Schaffer, Phys. Rev. B 100, 104511 (2019).

[25] N. T. Ziani, C. Fleckenstein, L. Vigliotti, B. Trauzettel, and M. Sassetti, Phys. Rev. B 101, 195303 (2020).

[26] J. Zhu, I. N. Krivorotov, K. Halterman, and O. T. Valls, Phys. Rev. Lett. 105, 207002 (2010).

[27] A. Di Bernardo, S. Diesch, Y. Gu, J. Linder, G. Divitini, C. Ducati, E. Scheer, M. G. Blamire, and J. W. Robinson, Nat. Commun. 6, 8053 (2015).

[28] A. Di Bernardo, Z. Salman, X. L. Wang, M. Amado, M. Egilmez, M. G. Flokstra, A. Suter, S. L. Lee, J. H. Zhao, T.
Prokscha, E. Morenzoni, M. G. Blamire, J. Linder, and J. W. A. Robinson, Phys. Rev. X 5, 041021 (2015).

[29] A. M. Black-Schaffer and A. V. Balatsky, Phys. Rev. B 88, 104514 (2013).

[30] F. Parhizgar and A. M. Black-Schaffer, Phys. Rev. B 90, 184517 (2014).

[31] B. Sothmann, S. Weiss, M. Governale, and J. König, Phys. Rev. B 90, 220501(R) (2014).

[32] L. Komendová, A. V. Balatsky, and A. M. Black-Schaffer, Phys. Rev. B 92, 094517 (2015).

[33] P. Burset, B. Lu, H. Ebisu, Y. Asano, and Y. Tanaka, Phys. Rev. B 93, 201402(R) (2016).

[34] H. Ebisu, B. Lu, J. Klinovaja, and Y. Tanaka, Prog. Theor. Exp. Phys. 2016, $083 \mathrm{I} 01$ (2016).

[35] J. Schmidt, F. Parhizgar, and A. M. Black-Schaffer, Phys. Rev. B 101, 180512(R) (2020).

[36] C. Triola and A. V. Balatsky, Phys. Rev. B 94, 094518 (2016).

[37] C. Triola and A. V. Balatsky, Phys. Rev. B 95, 224518 (2017).

[38] T. Papenkort, V. M. Axt, and T. Kuhn, Phys. Rev. B 76, 224522 (2007).

[39] H. Krull, D. Manske, G. S. Uhrig, and A. P. Schnyder, Phys. Rev. B 90, 014515 (2014).

[40] M. S. Rudner and N. H. Lindner, Nat. Rev. Phys. 2, 229 (2020).

[41] N. Kumar and K. P. Sinha, Phys. Rev. 174, 482 (1968).

[42] G. Nieva, E. Osquiguil, J. Guimpel, M. Maenhoudt, B. Wuyts, Y. Bruynseraede, M. B. Maple, and I. K. Schuller, Appl. Phys. Lett. 60, 2159 (1992).

[43] D. Fausti, R. Tobey, N. Dean, S. Kaiser, A. Dienst, M. C. Hoffmann, S. Pyon, T. Takayama, H. Takagi, and A. Cavalleri, Science 331, 189 (2011).

[44] M. A. Sentef, A. F. Kemper, A. Georges, and C. Kollath, Phys. Rev. B 93, 144506 (2016).

[45] M. Knap, M. Babadi, G. Refael, I. Martin, and E. Demler, Phys. Rev. B 94, 214504 (2016).

[46] Y. Wang, C.-C. Chen, B. Moritz, and T. P. Devereaux, Phys. Rev. Lett. 120, 246402 (2018).

[47] S. Porta, L. Privitera, N. T. Ziani, M. Sassetti, F. Cavaliere, and B. Trauzettel, Phys. Rev. B 100, 024513 (2019).

[48] M. Mitrano, A. Cantaluppi, D. Nicoletti, S. Kaiser, A. Perucchi, S. Lupi, P. Di Pietro, D. Pontiroli, M. Riccò, S. Clark et al., Nature 530, 461 (2016).

[49] A. M. Zagoskin, Quantum Theory of Many-Body Systems, Vol. 174 (Springer, Berlin-Heidelberg, 1998).

[50] G. D. Mahan, Many-Particle Physics (Springer, New York, 2013).

[51] G. Floquet, Annales scientifiques de l'École Normale Supérieure 12, 47 (1883).

[52] J. H. Shirley, Phys. Rev. 138, B979 (1965).

[53] H. Sambe, Phys. Rev. A 7, 2203 (1973).

[54] H. Aoki, N. Tsuji, M. Eckstein, M. Kollar, T. Oka, and P. Werner, Rev. Mod. Phys. 86, 779 (2014).

[55] See supplemental material at http://link.aps.org/supplemental/ 10.1103/PhysRevB.103.104505 for additional discussions to support the conclusions of this work. 
[56] Y. H. Wang, H. Steinberg, P. Jarillo-Herrero, and N. Gedik, Science 342, 453 (2013).

[57] J. W. McIver, B. Schulte, F.-U. Stein, T. Matsuyama, G. Jotzu, G. Meier, and A. Cavalleri, Nat. Phys. 16, 38 (2020).

[58] J. Shabani, M. Kjaergaard, H. J. Suominen, Y. Kim, F. Nichele, K. Pakrouski, T. Stankevic, R. M. Lutchyn, P. Krogstrup, R. Feidenhans'l, S. Kraemer, C. Nayak, M. Troyer, C. M. Marcus, and C. J. Palmstrøm, Phys. Rev. B 93, 155402 (2016).

[59] M. Kjaergaard, F. Nichele, H. J. Suominen, M. Nowak, M. Wimmer, A. R. Akhmerov, J. A. Folk, K. Flensberg, J. Shabani, C. J. Palmstrøm et al., Nat. Commun. 7, 12841 (2016).

[60] M. S. Rudner and N. H. Lindner, arXiv:2003.08252 (2020).

[61] F. Mahmood, C.-K. Chan, Z. Alpichshev, D. Gardner, Y. Lee, P. A. Lee, and N. Gedik, Nat. Phys. 12, 306 (2016).

[62] A detail study on the experimental consequences of these Floquet pair amplitudes will be presented elsewhere.

[63] Y. Tanaka, Y. Asano, A. A. Golubov, and S. Kashiwaya, Phys. Rev. B 72, 140503(R) (2005).

[64] Y. V. Fominov, Y. Tanaka, Y. Asano, and M. Eschrig, Phys. Rev. B 91, 144514 (2015).

[65] Y. Asano and A. Sasaki, Phys. Rev. B 92, 224508 (2015).

[66] J. A. Krieger, A. Pertsova, S. R. Giblin, M. Döbeli, T. Prokscha, C. W. Schneider, A. Suter, T. Hesjedal, A. V. Balatsky, and Z. Salman, Phys. Rev. Lett. 125, 026802 (2020).
[67] R. Shimano and N. Tsuji, Annu. Rev. Condens. Matter Phys. 11, 103 (2020).

[68] L. Schwarz, B. Fauseweh, N. Tsuji, N. Cheng, N. Bittner, H. Krull, M. Berciu, G. S. Uhrig, A. Schnyder, S. Kaiser et al., Nat. commun. 11, 287 (2020).

[69] G. Homann, J. G. Cosme, and L. Mathey, Phys. Rev. Research 2, 043214 (2020).

[70] A. A. Reynoso and D. Frustaglia, Phys. Rev. B 87, 115420 (2013).

[71] M. Benito, A. Gómez-León, V. M. Bastidas, T. Brandes, and G. Platero, Phys. Rev. B 90, 205127 (2014).

[72] A. Chew, D. F. Mross, and J. Alicea, Phys. Rev. Lett. 124, 096802 (2020).

[73] T. Oka and S. Kitamura, Annu. Rev. Condens. Matter Phys. 10, 387 (2019).

[74] U. D. Giovannini and H. Hübener, J. Phys. Mater. 3, 012001 (2019).

[75] S. Francesconi, F. Baboux, A. Raymond, N. Fabre, G. Boucher, A. Lemaitre, P. Milman, M. I. Amanti, and S. Ducci, Optica 7, 316 (2020).

[76] S. Porta, F. Cavaliere, M. Sassetti, and N. T. Ziani, Sci. Rep 10, 12766 (2020).

Correction: The copyright statement contained an error and has been fixed. 